\title{
Space charge effect in an accelerated beam
}

\author{
G. Stupakov and Z. Huang \\ Stanford Linear Accelerator Center, Stanford University, Stanford, California 94309, USA
}

(Received 22 August 2007; published 4 January 2008)

\begin{abstract}
It is usually assumed that the space charge effects in relativistic beams scale with the energy of the beam as $\gamma^{-2}$, where $\gamma$ is the relativistic factor. We show that for a beam accelerated in the longitudinal direction there is an additional space charge effect in free space that scales as $E / \gamma$, where $E$ is the accelerating field. This field has the same origin as the "electromagnetic mass of the electron" discussed in textbooks on electrodynamics. It keeps the balance between the kinetic energy of the beam and the energy of the electromagnetic field of the beam. We then consider the effect of this field on a beam generated in an rf gun and calculate the energy spread produced by this field in the beam.
\end{abstract}

DOI: 10.1103/PhysRevSTAB.11.014401

\section{INTRODUCTION}

Modern light sources such as free electron lasers and energy recovery linacs require high-peak current, smallemittance beams. One of the important characteristics of such a beam is its energy spread. It determines the limits of a possible bunch compression, the stability against microbunching, and properties of the beam as a radiator of photons. There are several mechanisms that contribute to the energy spread in radio frequency electron guns with the dominant one, for nanocoulomb bunches, being the space charge effect.

Traditionally in accelerator physics the space charge effect is computed as a self-field of a beam moving with constant velocity along a straight line. The longitudinal field in such a beam causes the energy exchange between the particles; it scales with the beam energy as $\gamma^{-2}$ [[1], page 128], where $\gamma$ is the relativistic factor, and usually becomes small for highly relativistic beams. In a broader sense, the space charge effect might be understood as a self-field of the beam, even when it moves with acceleration. With this understanding, acceleration adds to the beam self-field. One such contribution, which attracted a lot of attention lately, is due to the coherent radiation of the beam and is called the coherent synchrotron radiation wake (or CSR wake) [2-4]. The CSR wake is the radiation reaction force that keeps balance between the electromagnetic energy that is carried away by the radiation and the kinetic energy of the beam particles. It occurs when the beam is being accelerated in the direction perpendicular to the beam velocity in bending magnets or undulators.

Another type of radiation reaction force has been considered in recent papers $[5,6]$ - a self-field that arises inside the beam during a violent longitudinal acceleration in the direction of the beam velocity. This field is due to the radiation of the beam, however, it differs from CSR where the acceleration is perpendicular to the velocity. Such a field can play a role in plasma acceleration experiments, where the pace of acceleration is much larger than in a conventional rf cavities.
PACS numbers: 29.27.Bd, 29.25.Bx, 29.27.Eg, 41.85.Ja

In this paper we point out a new component of the space charge field that arises during a longitudinal acceleration of the beam. We assume that the acceleration is not strong enough to cause a noticeable radiation. During acceleration, however, the beam electromagnetic energy, which depends on the beam velocity, changes with time. To keep the balance between the beam and the field energy, one should expect an additional component of the selffield. The effect of this field, being proportional to the acceleration, on average is equivalent to a renormalization of the mass; it is discussed in textbooks on electrodynamics in connection with a so-called electromagnetic "mass" of a point charge $[7,8]$. In this paper we are interested in the spatial distribution of the field and, more specifically, the energy spread in the beam induced by the acceleration field.

The model that we consider in this paper assumes that the beam does not change its shape during the acceleration.

\section{ENERGY OF ELECTROMAGNETIC FIELD OF A MOVING GAUSSIAN BUNCH}

Consider a Gaussian bunch of charged particles moving with velocity $v$ in the $z$ direction with the particle distribution function given by

$$
n(x, y, \zeta)=\frac{N}{(2 \pi)^{3 / 2} \sigma_{z} \sigma_{\perp}^{2}} \exp \left(-\frac{r^{2}}{2 \sigma_{\perp}^{2}}-\frac{\zeta^{2}}{2 \sigma_{z}^{2}}\right)
$$

where $N$ is the number of particles in the bunch, $r=$ $\sqrt{x^{2}+y^{2}}, \zeta=z-v t, \sigma_{x}=\sigma_{y}=\sigma_{\perp}$ is the rms bunch size in the transverse direction, and $\sigma_{z}$ is the rms bunch length in the longitudinal direction. The electromagnetic field of such a bunch can be calculated using the Lorentz transformation from the beam frame, where the electrostatic potential is given by the following equation (see, e.g., [9]): 

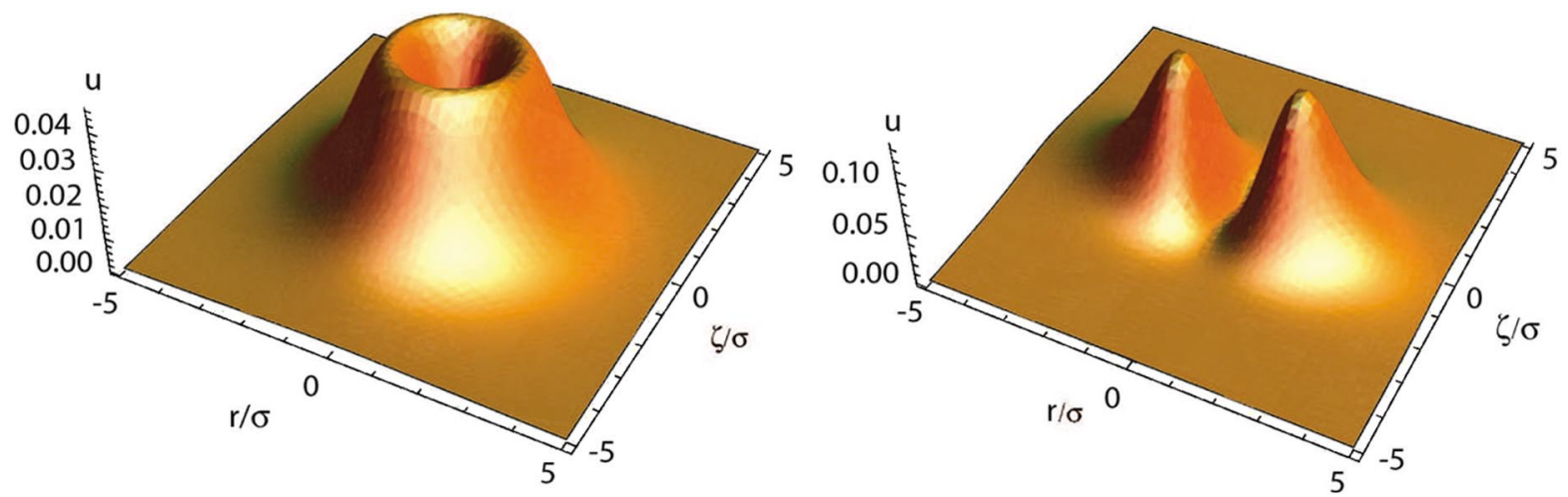

FIG. 1. (Color) Electromagnetic energy density $u$ in units $Q^{2} / 8 \pi \sigma^{4}$ for a spherical Gaussian bunch with (a) $\gamma=1$ and (b) $\gamma=2$. Note the difference in the vertical scales.

$$
\phi\left(x^{\prime}, y^{\prime}, z^{\prime}\right)=\sqrt{\frac{2}{\pi}} \frac{Q}{\sigma_{\perp}^{2} \sigma_{z}} \int_{0}^{\infty} e^{-\left[\left(x^{\prime 2}+y^{\prime 2}\right) \lambda^{2}\right] /\left[2\left(\lambda^{2} \sigma_{\perp}^{2}+1\right)\right]-\left(z^{\prime 2} \lambda^{2}\right) /\left[2\left(\lambda^{2} \tilde{\sigma}_{z}^{2}+1\right)\right]} \frac{d \lambda}{\left(\lambda^{2}+\sigma_{\perp}^{-2}\right) \sqrt{\lambda^{2}+\tilde{\sigma}_{z}^{-2}}},
$$

to the laboratory frame (we use the notation $Q=N e$ for the total charge of the bunch). In this equation $x^{\prime}, y^{\prime}, z^{\prime}$, and $\tilde{\sigma}_{z}=\gamma \sigma_{z}$ are the coordinates and the bunch length in the beam frame. For illustration, we plotted in Fig. 1 the energy density $u(r, \zeta)=\left(E^{2}+H^{2}\right) / 8 \pi$ of the electromagnetic field for a spherical Gaussian beam $\left(\sigma_{z}=\sigma_{\perp}=\sigma\right)$ at rest $(\gamma=1)$ and the same beam moving with relativistic factor $\gamma=2$. One can see that the energy density of the relativistically moving beam has a different spatial distribution and an increased magnitude. Correspondingly, the integrated electromagnetic energy over the whole space

$$
W=\int u d V
$$

grows with $\gamma$, as shown in Fig. 2. Note that this energy tends to infinity when $\gamma \rightarrow \infty$. As a detailed analysis shows, at $\gamma \gg 1$ the asymptotic expression for $W$ is

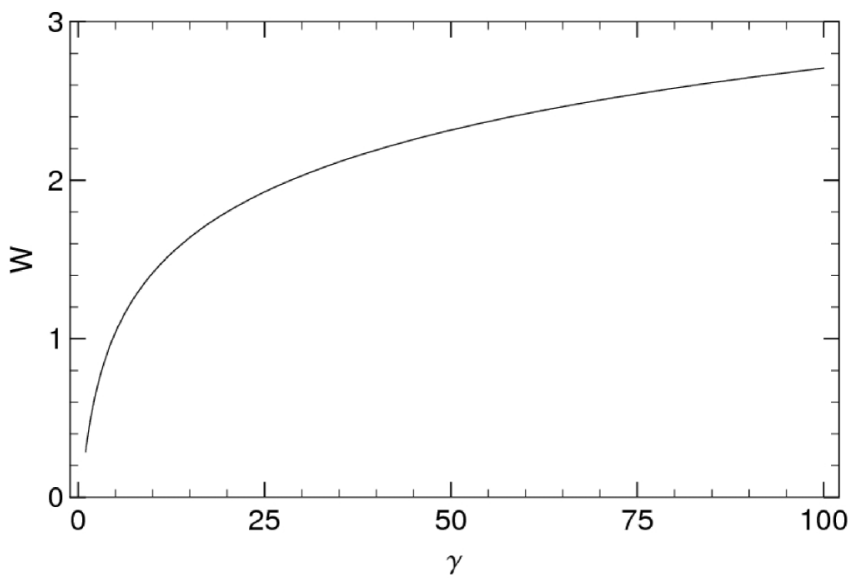

FIG. 2. Integrated electromagnetic energy density in units $Q^{2} / \sigma$ for a spherical Gaussian bunch as a function of $\gamma$.

$$
W=\frac{Q^{2}}{\sqrt{\pi} \sigma_{z}} \log \gamma, \quad \gamma \gg 1
$$

Imagine now that the beam is being accelerated from rest to velocity $v$ corresponding to some value of $\gamma$. The increased electromagnetic energy is taken from the kinetic energy of the beam via a longitudinal electric field $E_{z}$ induced by the acceleration. Such a field is known from the theory of the radiation reaction force [[8], page 386], where it is responsible for the electromagnetic field contribution to the mass of a charged particle. This force is linear in acceleration, and changes sign when the acceleration is reversed. It is not related to the radiation; in addition to transferring energy from the beam to the electromagnetic field during acceleration (and transferring it in the opposite direction during deceleration), it introduced an energy spread in the beam. In the next section we derive an expression for $E_{z}$ using the retarded potentials.

\section{SPACE CHARGE AND ACCELERATION FIELDS}

Consider a beam moving along the $z$ axis with velocity $v(t)$ that varies with time and is the same for all particles of the beam. If $n(x, y, z)$ is the particle density at initial time, then at time $t$ the charge density $\rho$ and the current density $j_{z}$ are $\rho=e n\left[x, y, z-z_{0}(t)\right], j_{z}=e v(t) n\left[x, y, z-z_{0}(t)\right]$, with $v=d z_{0} / d t$, and $e$ the elementary charge. The scalar and vector potentials of the beam are given by the following equations [7]:

$$
\begin{aligned}
\phi(\boldsymbol{r}, t) & =\int \frac{\rho\left(\boldsymbol{r}^{\prime}, t-\tau\right)}{|\boldsymbol{r}-\boldsymbol{r}|^{\prime}} d^{3} r^{\prime}, \\
\boldsymbol{A}\left(\boldsymbol{r}^{\prime}, t\right) & =\frac{1}{c} \int \frac{\boldsymbol{j}\left(\boldsymbol{r}^{\prime}, t-\tau\right)}{\left|\boldsymbol{r}-\boldsymbol{r}^{\prime}\right|} d^{3} r^{\prime},
\end{aligned}
$$


where the retarded time $t-\tau$ is defined by $c \tau=\left|\boldsymbol{r}-\boldsymbol{r}^{\prime}\right|$. We will assume that the acceleration $a(t)=d v / d t$ is small and expand the potentials in Taylor series keeping only linear terms in acceleration. We have approximately

$$
\begin{aligned}
z_{0}(t-\tau) & \approx z_{0}(t)-v(t) \tau+\frac{1}{2} a(t) \tau^{2}, \\
v(t-\tau) & \approx v(t)-a(t) \tau .
\end{aligned}
$$

This gives for the scalar potential

$$
\phi(\boldsymbol{r}, t)=e \int \frac{n\left[x^{\prime}, y^{\prime}, z^{\prime}-z_{0}(t)+v(t) \tau-\frac{1}{2} a(t) \tau^{2}\right]}{|\boldsymbol{r}-\boldsymbol{r}|^{\prime}} d^{3} r^{\prime} .
$$

We now expand the function $n$ to obtain

$$
\phi \approx \phi_{\mathrm{sc}}+\tilde{\phi}
$$

where $\phi_{\mathrm{sc}}$ is the space charge potential that does not depend on acceleration, and $\tilde{\phi}$ is a part of the potential proportional to the acceleration:

$$
\begin{aligned}
\phi_{\mathrm{sc}}(\boldsymbol{r}, t)= & e \int \frac{n\left[x^{\prime}, y^{\prime}, z^{\prime}-z_{0}(t)+v(t) \tau\right]}{|\boldsymbol{r}-\boldsymbol{r}|^{\prime}} d^{3} r^{\prime}, \\
\tilde{\phi}(\boldsymbol{r}, t)= & -\frac{e}{2} a(t) \int \frac{\tau^{2}}{|\boldsymbol{r}-\boldsymbol{r}|^{\prime}} \\
& \times \partial_{z} n\left[x^{\prime}, y^{\prime}, z^{\prime}-z_{0}(t)+v(t) \tau\right] d^{3} r^{\prime},
\end{aligned}
$$

with $\partial_{z} n=\partial n(x, y, z) / \partial z$. Similarly, we expand the vector potential $\boldsymbol{A}$ which has the $z$ component only,

$$
A_{z}(\boldsymbol{r}, t)=\frac{e}{c} \int \frac{[v(t)-a(t) \tau] n\left[x^{\prime}, y^{\prime}, z^{\prime}-z_{0}(t)+v(t) \tau-\frac{1}{2} a(t) \tau^{2}\right]}{|\boldsymbol{r}-\boldsymbol{r}|^{\prime}} d^{3} r^{\prime}
$$

to obtain

$$
A \approx A_{\mathrm{sc}}+\tilde{A}
$$

with

$$
\begin{aligned}
A_{z, \mathrm{sc}}(\boldsymbol{r}, t)= & \frac{e}{c} \int \frac{v(t) n\left[x^{\prime}, y^{\prime}, z^{\prime}-z_{0}(t)+v(t) \tau\right]}{|\boldsymbol{r}-\boldsymbol{r}|^{\prime}} d^{3} r^{\prime} \\
\tilde{A}_{z,}(\boldsymbol{r}, t)= & -\frac{e}{2 c^{3}} a(t) v(t) \int\left|\boldsymbol{r}-\boldsymbol{r}^{\prime}\right| \\
& \times \partial_{z} n\left[x^{\prime}, y^{\prime}, z^{\prime}-z_{0}(t)+v(t) \tau\right] d^{3} r^{\prime} \\
& -\frac{e a(t)}{c^{2}} \int n\left[x^{\prime}, y^{\prime}, z^{\prime}-z_{0}(t)+v(t) \tau\right] d^{3} r^{\prime} .
\end{aligned}
$$

One can formulate conditions of applicability of the approximations used above by requiring that the terms discarded in the Taylor expansions are small compared to those left. There are two such conditions,

$$
a \ll \frac{c^{2}}{l}, \quad\left|\frac{\dot{a}}{a}\right| \ll \frac{c}{l},
$$

where $l$ is the characteristic size of the bunch. These conditions mean that the acceleration is not large and does not change fast.

The electric field of the beam is a sum of the space charge field and a component that vanishes in the limit when $a=0$ :

$$
\boldsymbol{E} \approx \boldsymbol{E}_{\mathrm{sc}}+\tilde{\boldsymbol{E}}
$$

where $\boldsymbol{E}_{\mathrm{sc}}=-\nabla \boldsymbol{\phi}_{\mathrm{sc}}-c^{-1} \partial \boldsymbol{A}_{\mathrm{sc}} / \partial t$ and $\tilde{\boldsymbol{E}}=-\nabla \tilde{\boldsymbol{\phi}}-$ $c^{-1} \partial \tilde{\boldsymbol{A}} / \partial t$. The electric field $\boldsymbol{E}_{\mathrm{sc}}$ (and related to it the magnetic field $\boldsymbol{B}=\boldsymbol{v} \times \boldsymbol{E}_{\mathrm{sc}} / c$ ) is traditionally associated in accelerator physics with the space charge effect. The energy density and the total energy of this field is plotted in Figs. 1 and 2, respectively.
In this paper, we are interested in the electric field $\tilde{\boldsymbol{E}}$, and more specifically in the longitudinal component $\tilde{E}_{z}$,

$$
\tilde{E}_{z}=-\frac{\partial \tilde{\phi}}{\partial z}-\frac{1}{c} \frac{\partial \tilde{A}_{z}}{\partial t}
$$

which changes the kinetic energy of the beam particles. Using Eqs. (9) and (10) for calculation of $\tilde{E}_{z}$, we find that in addition to terms proportional to $a$ it also contains terms that involve $\dot{a}$ and $a^{2}$. We discard the latter as being small because of the conditions (13). This gives the following expression for $\tilde{E}_{z}$ :

$$
\begin{aligned}
\tilde{E}_{z}= & -\frac{e}{c^{2}} a \int \frac{n\left(x^{\prime}, y^{\prime}, z^{\prime}+v \tau\right)}{\left|\boldsymbol{r}-\boldsymbol{r}^{\prime}\right|} d^{3} r^{\prime} \\
& -\frac{e}{2 c^{2}} a \beta \int\left[\beta\left|\boldsymbol{r}-\boldsymbol{r}^{\prime}\right|-\left(z-z^{\prime}\right)\right] \\
& \times \partial_{z z} n\left(x^{\prime}, y^{\prime}, z^{\prime}+v \tau\right) d^{3} r^{\prime} \\
& +\frac{e}{2 c^{2}} a \int\left(\frac{z-z^{\prime}}{\left|\boldsymbol{r}-\boldsymbol{r}^{\prime}\right|}-4 \beta\right) \partial_{z} n\left(x^{\prime}, y^{\prime}, z^{\prime}+v \tau\right) d^{3} r^{\prime} .
\end{aligned}
$$

In this equation we set $t=z_{0}=0$ [which means that the density distribution of the beam at the observation time is now $n(x, y, z)]$, and suppressed the argument $t$ in $a$ and $v$. In what follows, for brevity, we call $\tilde{E}_{z}$ the acceleration field.

In the nonrelativistic case $\beta \rightarrow 0$ and $\gamma \rightarrow 1$, Eq. (16) reduces to ${ }^{1}$

${ }^{1}$ Comparing this expression with the terms proportional to acceleration in Eqs. (21)-(51) of Ref. [8], one finds that Eq. (17) has an extra term in the integrand. This seeming discrepancy is explained by the fact that the density $n$ in Eq. (17) is taken at a given observation time $t$, while the field in Eqs. (21)-(51) should be integrated with the density taken at the retarded time $t-\left|\boldsymbol{r}-\boldsymbol{r}^{\prime}\right| / c$. 


$$
\begin{aligned}
\tilde{E}_{z} \approx & -\frac{e}{c^{2}} a \int \frac{n\left(x^{\prime}, y^{\prime}, z^{\prime}\right)}{\left|\boldsymbol{r}-\boldsymbol{r}^{\prime}\right|} d^{3} r^{\prime} \\
& +\frac{e}{2 c^{2}} a \int \frac{z-z^{\prime}}{\left|\boldsymbol{r}-\boldsymbol{r}^{\prime}\right|} \partial_{z} n\left(x^{\prime}, y^{\prime}, z^{\prime}\right) d^{3} r^{\prime} \\
= & -\frac{e}{2 c^{2}} a \int\left[\frac{1}{\left|\boldsymbol{r}-\boldsymbol{r}^{\prime}\right|}+\frac{\left(z-z^{\prime}\right)^{2}}{\left|\boldsymbol{r}-\boldsymbol{r}^{\prime}\right|^{3}}\right] n\left(x^{\prime}, y^{\prime}, z^{\prime}\right) d^{3} r^{\prime} .
\end{aligned}
$$

\section{ACCELERATION FIELD FOR A GAUSSIAN BUNCH}

For a Gaussian bunch with the charge distribution function given by Eq. (1), the calculation of the acceleration field can be reduced to a one-dimensional integral. The expression for this field is derived in Appendix A and is given by Eqs. (A12)-(A14).

Using the relation between the acceleration and the rate of change of the gamma factor, $a=\left(c / \gamma^{3} \beta\right) d \gamma / d t$, we can write the energy change of a particle in the beam due to the acceleration field as

$$
\Delta \mathcal{E}(r, z)=\int v e \tilde{E}_{z} d t=-\frac{I_{0}}{I_{A}} m c^{2} \int_{\gamma_{i}}^{\gamma_{f}} \frac{d \gamma}{\gamma^{3}} G,
$$

where $I_{0}=N e c / \sqrt{2} \pi \sigma_{z}$ is the peak current in the bunch, $I_{A}=m c^{3} / e$ is the Alfvén current, $\gamma_{i}$ and $\gamma_{f}$ are the initial and final values of the gamma factor, and the function $G$ is given by Eq. (A13).

In the ultrarelativistic limit $\gamma \gg 1$, one can find from Eqs. (A13) and (A14) that $G \approx 2 \gamma^{2} e^{-z^{2} / 2 \sigma_{z}^{2}}$. The longitudinal acceleration field in this limit does not depend on radius. Taking into account that in the external field $E_{\text {ext }}$ the acceleration of a relativistic particle is $a=e E_{\text {ext }} / m \gamma^{3}$, we arrive at the following expression for $\tilde{E}_{z}$ :

$$
\tilde{E}_{z}=-\sqrt{\frac{2}{\pi}} \frac{r_{e} N}{\sigma_{z} \gamma} E_{\mathrm{ext}} e^{-z^{2} / 2 \sigma_{z}^{2}}, \quad \gamma \gg 1,
$$

with $r_{e}=e^{2} / m c^{2}$. We see that the acceleration field is directed against the external field $E_{\text {ext }}$ and scales as $E_{\text {ext }} / \gamma$. This contrasts to the usual scaling $\propto \gamma^{-2}$ of the longitudinal space charge forces. Note that due to the scaling $G \propto$ $\gamma^{2}$ the integral (18) diverges logarithmically when $\gamma_{f} \rightarrow$ $\infty$. This is related to the fact that the electromagnetic energy of the bunch logarithmically tends to infinity when $\gamma \rightarrow \infty$, as indicated by Eq. (4).

In reality, the beam is being accelerated inside a vacuum chamber that has a characteristic transverse size $b$. The presence of the metallic boundaries of the chamber changes the electromagnetic field of the beam: in addition to the free space field calculated above, there will be a field generated by image charges and currents in the wall. Calculation of the latter constitutes a much more difficult problem, which we do not intend in this paper. We note, however, that there are two important properties of the field of the image charges in the practically important case, when the size of the chamber is much larger than the dimensions of the beam, $b \gg \sigma_{z}, \sigma_{\perp}$. First, since these charges are located relatively far from the beam, variation of their field at the location of the beam over the distance $\sim \sigma$ is relatively small. This means that, with a good accuracy, calculation of the energy spread in the beam can be carried out using only the free space field. Second, the electromagnetic energy of a relativistic beam propagating in a pipe of radius $b$ does not change with $\gamma$ when $\gamma \gtrsim b / \sigma_{z}$. To take into account this shielding effect of the metallic pipe, for a rough estimates, we will assume that

$$
\gamma_{f}=\frac{b}{\sigma_{z}}
$$

Note that $\gamma_{f} \gg 1$ for $b / \sigma_{z} \gg 1$, and because our result has only a logarithmic dependence on $\gamma$, it is rather insensitive to the exact value of $\gamma_{f}$.

Taking into account the above considerations, for a numerical example, we consider now parameters of the Linac Coherent Light Source (LCLS) rf gun beam at SLAC. Because our model assumes Gaussian distribution and the LCLS beam has a flat longitudinal profile, we choose the model parameters in such a way that $\sigma_{x}$ and $\sigma_{z}$ are equal to the corresponding rms values for the LCLS beam. We have $\sigma_{z}=0.86 \mathrm{~mm}, \sigma_{x}=0.6 \mathrm{~mm}$, and $Q=$ $0.72 \mathrm{nC}$ (corresponding to the peak current of $I_{0}=$ $100 \mathrm{~A})$. We also choose $\gamma_{i}=1$ and $\gamma_{f}=20$, corresponding to the beam pipe radius of about $1.2 \mathrm{~cm}$. Using Eq. (18) we calculated the energy loss of each particle in the beam. The plot of the radial dependence of the function $\Delta \mathcal{E}(r, z)$ for several values of $z$ is shown in Fig. 3. The energy loss for the same beam averaged over the transverse coordinate is shown in Fig. 4 as a function of the position $z$. Finally,

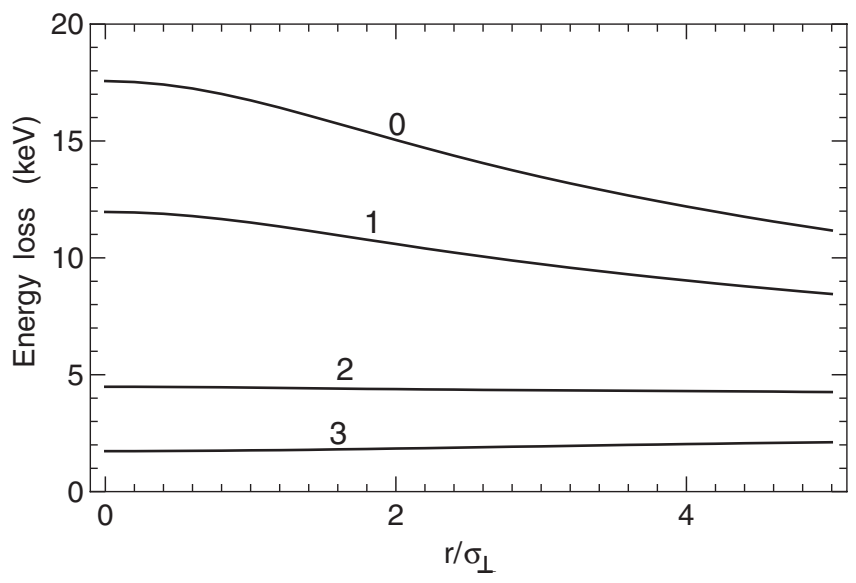

FIG. 3. Energy loss induced by the acceleration field for four different slices in the bunch $\left(z / \sigma_{z}=0,1,2\right.$, and 3 ; this number is indicated near the curves) as a function of electron radial position. 


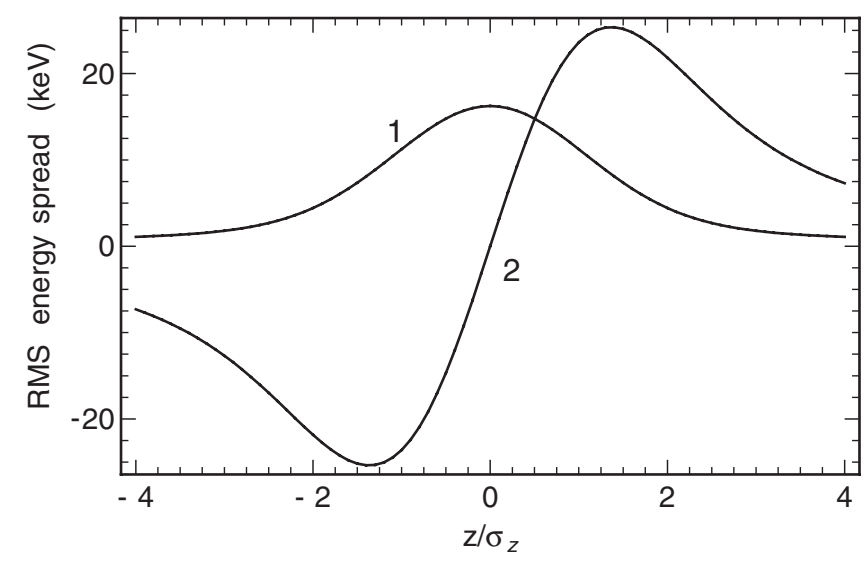

FIG. 4. Energy loss induced by the acceleration field (averaged over transverse dimensions) as a function of electron longitudinal position, curve 1 . For comparison, we also show the energy loss introduced by the space charge effect, curve 2 .

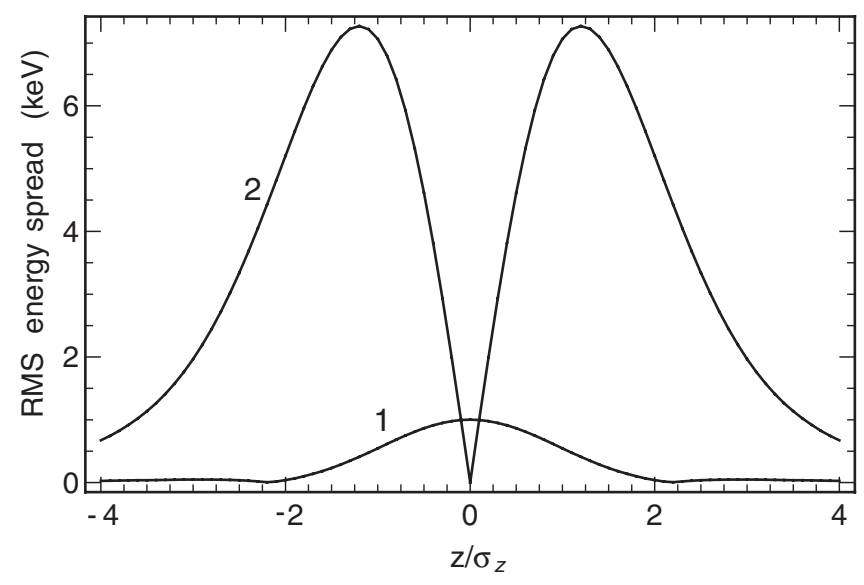

FIG. 5. Root-mean-square energy spread induced by the acceleration field as a function of electron longitudinal position, curve 1. For comparison, we also show the energy spread introduced by the space charge effect, curve 2 .

Fig. 5 shows the rms energy spread in slices as a function of $z$. In Figs. 3 and 4 we also show the energy loss and the rms energy spread introduced by the space charge forces (due to the longitudinal component of the field $\boldsymbol{E}_{\mathrm{sc}}$ ). The space charge effects were calculated using the theory of Ref. [10].

\section{DISCUSSION}

As mentioned in the Introduction, the acceleration field keeps balance between the electromagnetic energy of the beam and the kinetic energy of the particles. Mathematically, this property is formulated as the equality between the rate of change of the electromagnetic energy $W$ and the work of the field $\tilde{E}_{z}$ on the moving particles

$$
\frac{d W}{d t}=-e \int \tilde{E}_{z} v n d^{3} r .
$$

We present a proof of this statement in a nonrelativistic case $\beta \ll 1$ in Appendix B. Because of mathematical complexities, we were not able to prove it for an arbitrary, relativistic $\beta$; however, there is no doubt that it also holds in a general case. Within the formalism developed in this paper, this can be easily demonstrated by numerically calculating the left- and the right-hand side of Eq. (21). For illustration, we carried out such calculation for the parameters of the beam specified in the previous section. More specifically, we calculated the electromagnetic energy difference for the beam at the final state with $\gamma_{f}=20$ and the initial state with $\gamma_{i}=1$, which gave us $\Delta W=$ $22.5 \mu \mathrm{J}$. When we integrated the right side of Eq. (21) over time from the initial to the final state, we found that the work of the acceleration was numerically equal to $\Delta W$, in perfect agreement with the energy balance equation.

The model that we considered in this paper assumed that the beam does not change its shape during the acceleration. Such a model neglects a component of the self-field that is associated with the converging (or diverging) beams (see [11] and references therein). The field in this case is due to the fact that a converging beam changes its transverse dimensions with a corresponding change in the electromagnetic energy of the field that the beam carries with it.

Computation of beam self-fields is a critical aspect in numerical simulations of high-brightness electron beam generation. Many simulation codes (ASTRA, IMPACT, PARMELA) employ the quasistatic approximation and compute only the space charge fields. There exist a few codes that calculate the beam fields from the exact solutions of Maxwell's equations (i.e., the retarded potentials). A review of different computational approaches can be found in Ref. [12]. In this paper, starting with the retarded potentials and separating explicitly the self-fields into space charge and acceleration ones, we give an analytical expression for the longitudinal component of the acceleration field. Using a Gaussian bunch model and typical (LCLS) rf gun parameters, we calculate the energy spread introduced by the acceleration field and show that it gives rise to a small correction of the energy spread introduced by the space charge field. These results may be useful in guiding the simulation studies of high-brightness beams.

\section{ACKNOWLEDGMENTS}

This work was supported by the U.S. Department of Energy Contract No. DE-AC02-76SF00515.

\section{APPENDIX A: ACCELERATION FIELD FOR A GAUSSIAN BUNCH}

To eliminate the retarded argument $z^{\prime}+v \tau$ from the integrands in Eqs. (16) it is convenient to introduce the following variables: 


$$
\begin{aligned}
& \zeta^{\prime}=\gamma\left(z^{\prime}+\beta\left|\boldsymbol{r}-\boldsymbol{r}^{\prime}\right|\right), \quad \zeta=\gamma z, \\
& \boldsymbol{R}=(x, y, \zeta), \quad \boldsymbol{R}^{\prime}=\left(x^{\prime}, y^{\prime}, \zeta^{\prime}\right) .
\end{aligned}
$$

Using the relations

$$
\begin{aligned}
\frac{\partial \zeta^{\prime}}{\partial z^{\prime}} & =\gamma\left(1-\beta \frac{z-z^{\prime}}{|\boldsymbol{r}-\boldsymbol{r}|^{\prime}}\right)=\gamma \frac{|\boldsymbol{R}-\boldsymbol{R}|^{\prime}}{|\boldsymbol{r}-\boldsymbol{r}|^{\prime}} \\
\left|\boldsymbol{r}-\boldsymbol{r}^{\prime}\right| & =\gamma\left[\left|\boldsymbol{R}-\boldsymbol{R}^{\prime}\right|+\beta\left(\zeta-\zeta^{\prime}\right)\right] \\
z-z^{\prime} & =\gamma\left(\zeta-\zeta^{\prime}+\beta\left|\boldsymbol{R}-\boldsymbol{R}^{\prime}\right|\right),
\end{aligned}
$$

it is easy to show that

$$
\begin{aligned}
\int n\left(x^{\prime}, y^{\prime}, z^{\prime}+v \tau\right) \frac{d^{3} r^{\prime}}{|\boldsymbol{r}-\boldsymbol{r}|^{\prime}}= & \int n\left(x^{\prime}, y^{\prime}, \gamma^{-1} \zeta^{\prime}\right) \\
& \times \frac{d^{3} R^{\prime}}{\left|\boldsymbol{R}-\boldsymbol{R}^{\prime}\right|} .
\end{aligned}
$$

In this equation the longitudinal coordinate in function $n$ is now relativistically scaled with the factor $\gamma$ because of the Lorentz contraction, and the coordinate $z$ is equal to the $\zeta / \gamma$. For a beam moving with a constant velocity our change of variables is equivalent to making calculations in the beam frame and transforming the result back into the laboratory frame.

Using Eqs. (A2) and (A3), the field of Eq. (16) can be rewritten as

$$
\tilde{E}_{z}=\tilde{E}_{z 1}+\tilde{E}_{z 2}+\tilde{E}_{z 3},
$$

where

$$
\begin{aligned}
\tilde{E}_{z 1}= & -\frac{e}{c^{2}} a \int \frac{n\left(x^{\prime}, y^{\prime}, \gamma^{-1} \zeta^{\prime}\right)}{\left|\boldsymbol{R}-\boldsymbol{R}^{\prime}\right|} d^{3} R^{\prime}, \\
\tilde{E}_{z 2}= & \frac{e}{2 c^{2}} \frac{a \beta}{\gamma} \int\left(\zeta-\zeta^{\prime}\right) \partial_{z z} n\left(x^{\prime}, y^{\prime}, \gamma^{-1} \zeta^{\prime}\right) \frac{\left|\boldsymbol{r}-\boldsymbol{r}^{\prime}\right|}{\left|\boldsymbol{R}-\boldsymbol{R}^{\prime}\right|} d^{3} R^{\prime}, \\
\tilde{E}_{z 3}= & \frac{e}{2 c^{2}} a \int \frac{\gamma\left(\zeta-\zeta^{\prime}+\beta\left|\boldsymbol{R}-\boldsymbol{R}^{\prime}\right|-4 \beta\left|\boldsymbol{r}-\boldsymbol{r}^{\prime}\right|\right)}{\left|\boldsymbol{R}-\boldsymbol{R}^{\prime}\right|} \\
& \times \partial_{z} n\left(x^{\prime}, y^{\prime}, \gamma^{-1} \zeta^{\prime}\right) d^{3} R^{\prime} \\
= & \frac{e}{2 c^{2}} a \int\left[\frac{\gamma\left(1-4 \beta^{2}\right)\left(\zeta-\zeta^{\prime}\right)}{\left|\boldsymbol{R}-\boldsymbol{R}^{\prime}\right|}-3 \gamma \beta\right] \\
& \times \partial_{z} n\left(x^{\prime}, y^{\prime}, \gamma^{-1} \zeta^{\prime}\right) d^{3} R^{\prime} .
\end{aligned}
$$

We remind the reader that $\partial_{z} n\left(\partial_{z z} n\right)$ means first (second) derivative with respect to the third argument $z=\gamma^{-1} \zeta$ of the density function. Note also that the last term in $\tilde{E}_{z 3}$ integrates to zero because $\partial_{z} n=\gamma \partial n / \partial \zeta^{\prime}$.

Using Eq. (A2) and integrating by parts, we can simplify $\tilde{E}_{z 2}$ as

$$
\begin{aligned}
\tilde{E}_{z 2}= & \frac{e}{2 c^{2}} a \beta \gamma \int\left[\frac{\left(\zeta-\zeta^{\prime}\right)}{\gamma}+\frac{\beta\left(\zeta-\zeta^{\prime}\right)^{2}}{\gamma\left|\boldsymbol{R}-\boldsymbol{R}^{\prime}\right|}\right] \\
& \times n_{z z}\left(x^{\prime}, y^{\prime}, \gamma^{-1} \zeta^{\prime}\right) d^{3} R^{\prime} \\
= & \frac{e}{2 c^{2}} a \beta \gamma \int\left[1-\frac{\partial}{\partial\left(\gamma^{-1} \zeta^{\prime}\right)} \frac{\beta\left(\zeta-\zeta^{\prime}\right)^{2}}{\gamma\left|\boldsymbol{R}-\boldsymbol{R}^{\prime}\right|}\right] \\
& \times n_{z}\left(x^{\prime}, y^{\prime}, \gamma^{-1} \zeta^{\prime}\right) d^{3} R^{\prime} \\
= & -\frac{e}{2 c^{2}} a \beta^{2} \gamma \int\left[\frac{\left(\zeta-\zeta^{\prime}\right)^{3}}{\left|\boldsymbol{R}-\boldsymbol{R}^{\prime}\right|^{3}}-\frac{2\left(\zeta-\zeta^{\prime}\right)}{\left|\boldsymbol{R}-\boldsymbol{R}^{\prime}\right|}\right] \\
& \times n_{z}\left(x^{\prime}, y^{\prime}, \gamma^{-1} \zeta^{\prime}\right) d^{3} R^{\prime} .
\end{aligned}
$$

Thus, we have

$$
\begin{aligned}
\tilde{E}_{z 2}+\tilde{E}_{z 3}= & -\frac{e}{2 c^{2}} a \gamma \int\left[\frac{\beta^{2}\left(\zeta-\zeta^{\prime}\right)^{3}}{\left|\boldsymbol{R}-\boldsymbol{R}^{\prime}\right|^{3}}\right. \\
& \left.+\frac{\left(2 \beta^{2}-1\right)\left(\zeta-\zeta^{\prime}\right)}{\left|\boldsymbol{R}-\boldsymbol{R}^{\prime}\right|}\right] n_{z}\left(x^{\prime}, y^{\prime}, \gamma^{-1} \zeta^{\prime}\right) d^{3} R^{\prime} .
\end{aligned}
$$

Now we take a 3D Gaussian bunch distribution of Eq. (1) to obtain

$$
\begin{aligned}
\tilde{E}_{z 2}+\tilde{E}_{z 3}= & \frac{N e a}{2(2 \pi)^{3 / 2} c^{2} \sigma_{\perp}^{2} \sigma_{z}^{3}} \\
& \times \int d x^{\prime} d y^{\prime} \exp \left[-\frac{\left(x^{\prime}\right)^{2}+\left(y^{\prime}\right)^{2}}{2 \sigma_{\perp}^{2}}\right] \\
& \times \int d \zeta^{\prime} \zeta^{\prime} \exp \left[-\frac{\left(\zeta^{\prime}\right)^{2}}{2\left(\gamma \sigma_{z}\right)^{2}}\right] \\
& \times\left[\frac{\beta^{2}\left(\zeta-\zeta^{\prime}\right)^{3}}{\left|\boldsymbol{R}-\boldsymbol{R}^{\prime}\right|^{3}}+\frac{\left(2 \beta^{2}-1\right)\left(\zeta-\zeta^{\prime}\right)}{\left|\boldsymbol{R}-\boldsymbol{R}^{\prime}\right|}\right] .
\end{aligned}
$$

Using the relations

$$
\begin{aligned}
\sqrt{\frac{2}{\pi}} \int_{0}^{\infty} \exp \left(-\frac{\lambda^{2} r^{2}}{2}\right) d \lambda & =\frac{1}{r}, \\
\sqrt{\frac{2}{\pi}} \int_{0}^{\infty} \lambda^{2} \exp \left(-\frac{\lambda^{2} r^{2}}{2}\right) d \lambda & =\frac{1}{r^{3}},
\end{aligned}
$$

we replace the factors $\left|\boldsymbol{R}-\boldsymbol{R}^{\prime}\right|^{-1}$ and $\left|\boldsymbol{R}-\boldsymbol{R}^{\prime}\right|^{-3}$ in Eq. (A8) by integrals involving the integration over variable $\lambda$ :

$$
\begin{aligned}
\tilde{E}_{z 2}+\tilde{E}_{z 3}= & \frac{N e a}{4 \pi^{2} c^{2} \sigma_{\perp}^{2} \sigma_{z}^{3}} \int d x^{\prime} d y^{\prime} \exp \left[-\frac{\left(x^{\prime}\right)^{2}+\left(y^{\prime}\right)^{2}}{2 \sigma_{\perp}^{2}}\right] \\
& \times \int d \zeta^{\prime} \zeta^{\prime} \exp \left[-\frac{\left(\zeta^{\prime}\right)^{2}}{2\left(\gamma \sigma_{z}\right)^{2}}\right] \\
& \times \int_{0}^{\infty} d \lambda\left[\beta^{2} \lambda^{2}\left(\zeta-\zeta^{\prime}\right)^{3}+\left(2 \beta^{2}-1\right)\left(\zeta-\zeta^{\prime}\right)\right] \\
& \times \exp \left(-\frac{\lambda^{2}\left|\boldsymbol{R}-\boldsymbol{R}^{\prime}\right|^{2}}{2}\right) .
\end{aligned}
$$

Analogously, for the field $\tilde{E}_{z 1}$ we find 


$$
\begin{aligned}
\tilde{E}_{z 1}= & -\frac{N e a}{2 \pi^{2} c^{2} \sigma_{\perp}^{2} \sigma_{z}} \int d x^{\prime} d y^{\prime} \exp \left[-\frac{\left(x^{\prime}\right)^{2}+\left(y^{\prime}\right)^{2}}{2 \sigma_{\perp}^{2}}\right] \\
& \times \int d \zeta^{\prime} \exp \left[-\frac{\left(\zeta^{\prime}\right)^{2}}{2\left(\gamma \sigma_{z}\right)^{2}}\right] \\
& \times \int_{0}^{\infty} d \lambda \exp \left(-\frac{\lambda^{2}\left|\boldsymbol{R}-\boldsymbol{R}^{\prime}\right|^{2}}{2}\right) .
\end{aligned}
$$

If we change the integration variable to $\xi=\left(\lambda \sigma_{z} \gamma\right)^{-2}$ and define the bunch aspect ratio as $A=\sigma_{\perp} / \sigma_{z}$, then the result of integration is

$$
\tilde{E}_{z}(x, y, z)=-\frac{e N a}{\sqrt{2 \pi} c^{2} \sigma_{z}} G\left(\frac{r}{\sigma_{\perp}}, \frac{z}{\sigma_{z}}\right),
$$

with

$G(R, Z)=\int_{0}^{\infty} d \xi \exp \left[-\frac{R^{2} A^{2}}{2\left(A^{2}+\xi \gamma^{2}\right)}-\frac{Z^{2}}{2(\xi+1)}\right] F(\xi, Z)$,

where

$$
\begin{aligned}
F(\xi, Z)= & \frac{\gamma^{2}}{2(\xi+1)^{9 / 2}\left(A^{2}+\gamma^{2} \xi\right)} \\
& \times\left(\xi \left\{5 Z^{2}+\left[-\xi Z^{4}+2(\xi-2)(\xi+1) Z^{2}\right.\right.\right. \\
& \left.\left.+\xi(\xi+3)^{2}\right] \gamma^{2}\right\} \\
& \left.+\xi\left\{4 \gamma^{2}+\xi\left[Z^{4}-(\xi-4) Z^{2}-\xi\right]+3\right\}+2\right) .
\end{aligned}
$$

\section{APPENDIX B: ENERGY CONSERVATION AND THE ACCELERATION FIELD}

In this section we will prove that the work of the acceleration field is equal to the change of the electromagnetic energy of the bunch in the course of acceleration. Our proof is only valid for nonrelativistic velocities, $v \ll c$.

Let us assume that the beam, initially at $t=0$ at rest, is accelerated to velocity $v$ at time $t$. The work $\mathcal{A}$ done by the acceleration field is

$$
\begin{aligned}
\mathcal{A}= & -e \int_{0}^{t} d t^{\prime} \boldsymbol{v}\left(t^{\prime}\right) \int n\left(\boldsymbol{r}, t^{\prime}\right) \tilde{E}_{z}\left(\boldsymbol{r}, t^{\prime}\right) d^{3} r \\
= & \frac{e^{2}}{2 c^{2}} \int_{0}^{t} d t^{\prime} v\left(t^{\prime}\right) a\left(t^{\prime}\right) \int n\left(\boldsymbol{r}, t^{\prime}\right) n\left(\boldsymbol{r}, t^{\prime}\right) \\
& \times\left[\frac{1}{\left|\boldsymbol{r}-\boldsymbol{r}^{\prime}\right|}+\frac{\left(z-z^{\prime}\right)^{2}}{\left|\boldsymbol{r}-\boldsymbol{r}^{\prime}\right|^{3}}\right] d^{3} r^{\prime} d^{3} r \\
= & \frac{e^{2} \beta^{2}}{4} \int n(\boldsymbol{r}) n\left(\boldsymbol{r}^{\prime}\right)\left[\frac{1}{\left|\boldsymbol{r}-\boldsymbol{r}^{\prime}\right|}+\frac{\left(z-z^{\prime}\right)^{2}}{\left|\boldsymbol{r}-\boldsymbol{r}^{\prime}\right|^{3}}\right] d^{3} r^{\prime} d^{3} r,
\end{aligned}
$$

where $\beta=v(t) / c$, and we used Eq. (17) for the field $\tilde{E}_{z}$. Note that the time dependence of the density distribution $n(\boldsymbol{r}, t)$ reduces to a mere shift of the initial $n$ to the position of the bunch at time $t$. We eliminate this shift on the last line of Eq. (B1) by assuming that the coordinate system at any time $t$ is positioned in such a way that the center of the bunch is always located at the origin of the coordinate system [note that this is also assumed in Eq. (17)]. We will use such coordinates in the forthcoming calculations of the electromagnetic energy as well.

To calculate the change of the electromagnetic energy $\Delta W$ when the beam is accelerated from the initial to the final state, $\Delta W=W_{f}-W_{i}$, we use the expressions for the energy of the electromagnetic field

$$
W_{i}=\frac{1}{8 \pi} \int d^{3} r E_{i}^{2}, \quad W_{f}=\frac{1}{8 \pi} \int d^{3} r\left(E_{f}^{2}+B_{f}^{2}\right),
$$

where in the first expression for $W_{i}$ we took into account that the magnetic field of the beam at rest is equal to zero.

We now note that for $\beta \ll 1$, as it follows from Lorentz transformations for the fields, $B_{f} \approx \beta E_{i \perp}$, and also $\boldsymbol{E}_{f}=$ $\boldsymbol{E}_{i}+\delta \boldsymbol{E}$ with $\delta E$ being of the order of $\beta^{2} E_{i}$. Also note that, since we assume that at the initial state the beam is at rest, the electric field $\boldsymbol{E}_{i}$ can be expressed as a gradient of the electrostatic potential, $\boldsymbol{E}_{i}=-\nabla \phi$. We will now prove that the contribution of the electric field to $\Delta W$ vanishes. This contribution is

$$
\begin{aligned}
\frac{1}{8 \pi} \int d^{3} r\left(E_{f}^{2}-E_{i}^{2}\right) & \approx \frac{1}{4 \pi} \int d^{3} r \boldsymbol{E}_{i} \cdot \boldsymbol{\delta} \boldsymbol{E} \\
& =-\frac{1}{4 \pi} \int d^{3} r \nabla \phi \cdot \boldsymbol{\delta} \boldsymbol{E} \\
& =\frac{1}{4 \pi} \int d^{3} r \phi(\nabla \cdot \boldsymbol{\delta} \boldsymbol{E}) .
\end{aligned}
$$

The last equation in this expression is equal to zero because $\nabla \cdot \boldsymbol{\delta} \boldsymbol{E}=\nabla \cdot \boldsymbol{E}_{f}-\nabla \cdot \boldsymbol{E}_{f}$ and both fields satisfy $\nabla \cdot$ $\boldsymbol{E}_{f}=\nabla \cdot \boldsymbol{E}_{i}=4 \pi e n$. Hence, the only contribution to the energy change comes from the magnetic field

$$
\begin{aligned}
\Delta W & =\frac{1}{8 \pi} \int d^{3} r B_{f}^{2}=\frac{\beta^{2}}{8 \pi} \int d^{3} r E_{i \perp}^{2} \\
& =\frac{\beta^{2}}{8 \pi} \int d^{3} r\left[\left(\partial_{x} \phi\right)^{2}+\left(\partial_{y} \phi\right)^{2}\right] .
\end{aligned}
$$

We now use the following expression for the electrostatic potential:

$$
\phi(\boldsymbol{r})=e \int d^{3} r^{\prime} \frac{n\left(\boldsymbol{r}^{\prime}\right)}{\left|\boldsymbol{r}-\boldsymbol{r}^{\prime}\right|},
$$

which gives for $\Delta W$

$$
\begin{aligned}
\Delta W= & \frac{\beta^{2} e^{2}}{8 \pi} \int d^{3} r d^{3} r^{\prime} d^{3} r^{\prime \prime} n\left(\boldsymbol{r}^{\prime}\right) n\left(\boldsymbol{r}^{\prime \prime}\right)\left(\frac{\partial\left|\boldsymbol{r}-\boldsymbol{r}^{\prime}\right|^{-1}}{\partial x}\right. \\
& \left.\times \frac{\partial\left|\boldsymbol{r}-\boldsymbol{r}^{\prime \prime}\right|^{-1}}{\partial x}+\frac{\partial\left|\boldsymbol{r}-\boldsymbol{r}^{\prime}\right|^{-1}}{\partial y} \frac{\partial\left|\boldsymbol{r}-\boldsymbol{r}^{\prime \prime}\right|^{-1}}{\partial y}\right) .
\end{aligned}
$$

To calculate this integral we consider the following tensor: 


$$
T_{\alpha \beta}=\int d^{3} r \frac{\partial\left|\boldsymbol{r}-\boldsymbol{r}^{\prime}\right|^{-1}}{\partial x_{\alpha}} \frac{\partial\left|\boldsymbol{r}-\boldsymbol{r}^{\prime \prime}\right|^{-1}}{\partial x_{\beta}},
$$

where the indices $\alpha$ and $\beta$ take values 1,2, and 3 with $x_{1}$, $x_{2}$, and $x_{3}$ associated with the coordinates $x, y$, and $z$, respectively. From the symmetry arguments it follows that $T_{\alpha \beta}$ is a function of the difference $\boldsymbol{\rho}=\boldsymbol{r}^{\prime}-\boldsymbol{r}^{\prime \prime}$. A general form of such a tensor is

$$
T_{\alpha \beta}=L(\rho) \delta_{\alpha \beta} \frac{1}{\rho}+M(\rho) \frac{\rho_{\alpha} \rho_{\beta}}{\rho^{3}},
$$

where $L$ and $M$ are yet unknown functions of the magnitude $\rho$ of the vector $\boldsymbol{\rho}$. To find these functions, we first compute the quantity $T_{\alpha \alpha}=(3 L+M) / \rho$ (as always, we assume summation over repeated indices). Using integration by parts and the relation $\partial^{2}|\boldsymbol{r}|^{-1} / \partial x_{\alpha} \partial x_{\alpha}=-4 \pi \delta(\boldsymbol{r})$, we find from Eq. (B6)

$$
\begin{aligned}
T_{\alpha \alpha} & =\int d^{3} r \frac{\partial\left|\boldsymbol{r}-\boldsymbol{r}^{\prime}\right|^{-1}}{\partial x_{\alpha}} \frac{\partial\left|\boldsymbol{r}-\boldsymbol{r}^{\prime \prime}\right|^{-1}}{\partial x_{\alpha}} \\
& =-\int d^{3} r \frac{1}{\left|\boldsymbol{r}-\boldsymbol{r}^{\prime}\right|} \frac{\partial^{2}\left|\boldsymbol{r}-\boldsymbol{r}^{\prime \prime}\right|^{-1}}{\partial x_{\alpha} \partial x_{\alpha}} \\
& =4 \pi \int d^{3} r \frac{\delta\left(\boldsymbol{r}-\boldsymbol{r}^{\prime \prime}\right)}{\left|\boldsymbol{r}-\boldsymbol{r}^{\prime}\right|}=\frac{4 \pi}{\rho} .
\end{aligned}
$$

Hence,

$$
3 L+M=4 \pi .
$$

The second equation for $L$ and $M$ is obtained from calculation of the quantity $\partial T_{\alpha \beta} / \partial \rho_{\beta}$. As follows from Eq. (B7), $\partial T_{\alpha \beta} / \partial \rho_{\beta}=(M-L) \rho_{\alpha} / \rho^{3}$. Using Eq. (B6) and replacing $\partial / \partial \rho_{\beta} \rightarrow-\partial / \partial x^{\prime \prime}{ }_{\beta}$ we find

$$
\begin{aligned}
\frac{\partial T_{\alpha \beta}}{\partial \rho_{\beta}} & =-\frac{\partial}{\partial x_{\beta}^{\prime \prime}} \int d^{3} r \frac{\partial\left|\boldsymbol{r}-\boldsymbol{r}^{\prime}\right|^{-1}}{\partial x_{\alpha}} \frac{\partial\left|\boldsymbol{r}-\boldsymbol{r}^{\prime \prime}\right|^{-1}}{\partial x_{\beta}} \\
& =\int d^{3} r \frac{\partial\left|\boldsymbol{r}-\boldsymbol{r}^{\prime}\right|^{-1}}{\partial x_{\alpha}} \frac{\partial^{2}\left|\boldsymbol{r}-\boldsymbol{r}^{\prime \prime}\right|^{-1}}{\partial x_{\beta}^{\prime \prime} \partial x_{\beta}^{\prime \prime}} \\
& =4 \pi \int d^{3} r \delta\left(\boldsymbol{r}-\boldsymbol{r}^{\prime \prime}\right) \frac{x_{\alpha}-x_{\alpha}^{\prime}}{\left|\boldsymbol{r}-\boldsymbol{r}^{\prime}\right|^{3}}=-\frac{4 \pi \rho_{\alpha}}{\rho^{3}},
\end{aligned}
$$

which gives

$$
L-M=4 \pi .
$$

From Eqs. (B9) and (B11) we now find

$$
L=-M=2 \pi \text {. }
$$

Using Eqs. (B6) and (B7) we find

$$
\begin{aligned}
& \int d^{3} r\left(\frac{\partial\left|\boldsymbol{r}-\boldsymbol{r}^{\prime}\right|^{-1}}{\partial x} \frac{\partial\left|\boldsymbol{r}-\boldsymbol{r}^{\prime \prime}\right|^{-1}}{\partial x}+\frac{\partial\left|\boldsymbol{r}-\boldsymbol{r}^{\prime}\right|^{-1}}{\partial y}\right. \\
& \left.\times \frac{\partial\left|\boldsymbol{r}-\boldsymbol{r}^{\prime \prime}\right|^{-1}}{\partial y}\right) \\
& =\frac{4 \pi}{\left|\boldsymbol{r}^{\prime}-\boldsymbol{r}^{\prime \prime}\right|}-2 \pi \frac{\left(x^{\prime}-x^{\prime \prime}\right)^{2}+\left(y^{\prime}-y^{\prime \prime}\right)^{2}}{\left|\boldsymbol{r}^{\prime}-\boldsymbol{r}^{\prime \prime}\right|^{3}} \\
& =\frac{2 \pi}{\left|\boldsymbol{r}^{\prime}-\boldsymbol{r}^{\prime \prime}\right|}\left(1+\frac{\left(z^{\prime}-z^{\prime \prime}\right)^{2}}{\left|\boldsymbol{r}^{\prime}-\boldsymbol{r}^{\prime \prime}\right|^{2}}\right) .
\end{aligned}
$$

Substituting this equality into Eq. (B5) we obtain

$$
\begin{aligned}
\Delta W= & \frac{\beta^{2} e^{2}}{4} \int d^{3} r^{\prime} d^{3} r^{\prime \prime} n\left(\boldsymbol{r}^{\prime}\right) n\left(\boldsymbol{r}^{\prime \prime}\right) \frac{1}{\left|\boldsymbol{r}^{\prime}-\boldsymbol{r}^{\prime \prime}\right|} \\
& \times\left(1+\frac{\left(z^{\prime}-z^{\prime \prime}\right)^{2}}{\left|\boldsymbol{r}^{\prime}-\boldsymbol{r}^{\prime \prime}\right|^{2}}\right),
\end{aligned}
$$

which, upon comparing with Eq. (B1), proves that $\Delta W=$ $\mathcal{A}$.

[1] A. W. Chao and M. Tigner, Handbook of Accelerator Physics and Engineering (World Scientific, Singapore, 2006), 3rd ed.

[2] L. V. Iogansen and M. S. Rabinovich, Sov. Phys. JETP 37, 83 (1960).

[3] Y.S. Derbenev, J. Rossbach, E. L. Saldin, and V.D. Shiltsev, DESY FEL Report TESLA-FEL 95-05, Deutsches Elektronen-Synchrotron, Hamburg, Germany, 1995.

[4] J. B. Murphy, S. Krinsky, and R. L. Gluckstern, Part. Accel. 57, 9 (1997).

[5] G. Geloni, E. Saldin, E. Schneidmiller, and M. Yurkov, Nucl. Instrum. Methods Phys. Res., Sect. A 578, 34 (2007).

[6] R. A. Bosch, Phys. Rev. ST Accel. Beams 10, 050701 (2007).

[7] J.D. Jackson, Classical Electrodynamics (Wiley, New York, 1999), 3rd ed.

[8] W. Panofsky and M. Phillips, Classical Electricity and Magnetism (Addison-Wesley, Reading, MA, 1962), 2nd ed.

[9] G. Stupakov, Lecture notes on Classical Mechanics and Electromagnetism in Accelerator Physics (The US Particle Accelerator School, Lansing, MI, 2007).

[10] Z. Huang, D. Dowell, P. Emma, C. Limborg-Deprey, G. Stupakov, and J. Wu, in Proceedings of the 2005 Particle Accelerator Conference, Knoxville, Tennessee USA, 2005, p. TPAT062.

[11] K. L. F. Bane and A. W. Chao, Phys. Rev. ST Accel. Beams 5, 104401 (2002).

[12] L. Serafini, in Proceedings of the 2nd ICFA Workshop on the Physics of High Brightness Beams, 1999, p. 27. 